\title{
Extending an Almost Complete Pair of Matrices to a Complete Triple
}

\author{
Sheng Chen and Chao Xia \\ Department of Mathematics, Harbin Institute of Technology, Harbin 150001, China \\ Correspondence should be addressed to Sheng Chen; schenhit@gmail.com
}

Received 18 February 2014; Accepted 3 April 2014; Published 27 April 2014

Academic Editor: Lingju Kong

Copyright (C) 2014 S. Chen and C. Xia. This is an open access article distributed under the Creative Commons Attribution License, which permits unrestricted use, distribution, and reproduction in any medium, provided the original work is properly cited.

Motivated by the concept of complete pairs, which was introduced by Krein and Langer, we present the concepts of an almost complete pair of matrices and a complete triple of matrices. It is proved that an almost complete pair of matrices can be extended to a complete triple. An application of the result to differential equations is also given.

\section{Introduction}

Let

$$
L(\lambda)=I \lambda^{l}+A_{l-1} \lambda^{l-1}+\cdots+A_{0}
$$

be a monic matrix polynomial, where $A_{i} \in M_{n}(\mathbb{C})$ for $i=$ $0,1, \ldots, l-1$. If there exist a matrix $S$ and a polynomial $L_{1}(\lambda)$ of degree $l-1$ such that $L(\lambda)=L_{1}(\lambda)(I \lambda-S)$, then $I \lambda-S$ is said to be a monic right divisor of $L(\lambda)$. As is well known, $I \lambda-S$ is a monic right divisor of $L(\lambda)$ if and only if $S$ is a root of $L(\lambda)$; that is,

$$
S^{l}+A_{l-1} S^{l-1}+\cdots+A_{0}=0 .
$$

Let $C$ be the companion matrix of $L(\lambda)$; that is,

$$
C=\left(\begin{array}{ccccc}
0 & I & 0 & \cdots & 0 \\
0 & 0 & I & \cdots & 0 \\
\vdots & \vdots & \vdots & & \vdots \\
0 & 0 & 0 & \cdots & I \\
-A_{0} & -A_{1} & -A_{2} & \cdots & -A_{l-1}
\end{array}\right)
$$

Then we have $\operatorname{det}(I \lambda-C)=\operatorname{det}(L(\lambda))$. If $S_{1}, S_{2}, \ldots, S_{l} \in$ $M_{n}(\mathbb{C})$ are the different $l$ roots of $L(\lambda)$, it is clear that the Vandermonde matrix

$$
W\left(S_{1}, S_{2}, \ldots, S_{l}\right)=\left(\begin{array}{ccccc}
I & I & I & \cdots & I \\
S_{1} & S_{2} & S_{3} & \cdots & S_{l} \\
S_{1}^{2} & S_{2}^{2} & S_{3}^{2} & \cdots & S_{l}^{2} \\
\vdots & \vdots & \vdots & & \vdots \\
S_{1}^{l-1} & S_{2}^{l-1} & S_{3}^{l-1} & \cdots & S_{l}^{l-1}
\end{array}\right)
$$

plays a role in the investigation of the spectral analysis of $L(\lambda)$ [1].

Definition 1. We say that the $l$-tuple $\left(S_{1}, S_{2}, \ldots, S_{l}\right)$ of complex square matrices of order $n$ is complete if the Vandermonde matrix $W\left(S_{1}, S_{2}, \ldots, S_{l}\right)$ is invertible. When $l=2$ and $l=3$, we call a complete $l$-tuple of matrices a complete pair and a complete triple, respectively.

Suppose that $\left(S_{1}, S_{2}\right)$ is complete. Take $P=S_{2}-S_{1}$. Then $P$ is invertible. If we take

$$
\begin{aligned}
A_{1} & =-P S_{2} P^{-1}-S_{1}, \quad A_{2}=P S_{2} P^{-1} S_{1}, \\
L(\lambda) & =I \lambda^{2}+A_{1} \lambda+A_{2},
\end{aligned}
$$

then $I \lambda-S_{1}, I \lambda-S_{2}$, form a complete pair of right divisors of $L(\lambda)$ (cf. Lemma 2.4 in [2]). The concept of complete pair was 
introduced in [3, 4]. In [5], Lancaster gave some important applications of complete pairs to solutions of differential equations. Motivated by the results in [5], we are interested in the following question.

Question 1. Suppose that $\left(S_{1}, S_{2}\right)$ is not a complete pair. Is it possible for us to find $S_{3}$ and a cubic matrix polynomial $L(\lambda)$ such that $\left(S_{1}, S_{2}, S_{3}\right)$ is a complete triple and

$$
I \lambda-S_{1}, \quad I \lambda-S_{2}, \quad I \lambda-S_{3}
$$

are right divisors of $L(\lambda)$ ?

If $\left(S_{1}, S_{2}, S_{3}\right)$ is a complete triple, then

$$
\left(\begin{array}{cc}
I & I \\
S_{1} & S_{2} \\
S_{1}^{2} & S_{2}^{2}
\end{array}\right)
$$

is of full column rank. Note that if $S_{1}, S_{2} \in M_{n}(\mathbb{C})$ and the rank of $S_{1}-S_{2}$ is less than $n-1$, we cannot always find $S_{3}$ such that $\left(S_{1}, S_{2}, S_{3}\right)$ is a complete triple. For example, let $S_{1}, S_{2} \in$ $M_{n}(\mathbb{C})$ be defined as follows:

$$
S_{1}=\left(\begin{array}{cc}
A_{1} & \alpha \\
\beta & A_{2}
\end{array}\right),
$$

where $A_{1}, A_{2}$ are square matrices of orders $n-2$ and 2, respectively, and $S_{2}-S_{1}$ is in the form

$$
\left(\begin{array}{ccc}
I_{n-2} & 0 & 0 \\
0 & 0 & 0 \\
0 & 0 & 0
\end{array}\right) .
$$

Then

$$
S_{2}^{2}-S_{1}^{2}=\left(\begin{array}{cc}
2 A_{1}+I_{n-2} & \alpha \\
\beta & 0
\end{array}\right)
$$

If $\operatorname{rank}(\alpha)=1$, then the last two columns of $\left(\begin{array}{c}S_{2}-S_{1} \\ S_{2}^{2}-S_{1}^{2}\end{array}\right)$ are linearly dependent. For any $S_{3} \in M_{n}(\mathbb{C})$,

$$
\left(\begin{array}{ccc}
I & I & I \\
S_{1} & S_{2} & S_{3} \\
S_{1}^{2} & S_{2}^{2} & S_{3}^{2}
\end{array}\right)
$$

cannot be invertible.

If $\operatorname{rank}(\alpha)=2$, then

$$
\left(\begin{array}{cc}
I & I \\
S_{1} & S_{2} \\
S_{1}^{2} & S_{2}^{2}
\end{array}\right)
$$

is of full column rank. From the condition that

$$
\left(\begin{array}{cc}
I & I \\
S_{1} & S_{2} \\
S_{1}^{2} & S_{2}^{2}
\end{array}\right)
$$

is of full column rank, we cannot conclude that $\operatorname{rank}\left(S_{2}-S_{1}\right) \geq$ $n-1$.

After the above discussion, we give the following definition.

Definition 2. For two matrices $S_{1}, S_{2} \in M_{n}(\mathbb{C})$, one says they form an almost complete pair if the matrix

$$
\left(\begin{array}{cc}
I & I \\
S_{1} & S_{2} \\
S_{1}^{2} & S_{2}^{2}
\end{array}\right)
$$

is of full column rank and the rank of $S_{1}-S_{2}$ is $n-1$. For simplification, one says $\left(S_{1}, S_{2}\right)$ is an almost complete pair of matrices of order $n$.

The following is the main result of this paper.

Theorem 3. Suppose that $S_{1}, S_{2} \in M_{n}(\mathbb{C}), n \geq 2$, and $\left(S_{1}, S_{2}\right)$ is an almost complete pair. Then one can find a matrix $S_{3} \in$ $M_{n}(\mathbb{C})$ such that $\left(S_{1}, S_{2}, S_{3}\right)$ is a complete triple; that is,

$$
\left(\begin{array}{ccc}
I & I & I \\
S_{1} & S_{2} & S_{3} \\
S_{1}^{2} & S_{2}^{2} & S_{3}^{2}
\end{array}\right)
$$

is invertible.

We prove this theorem in Section 2. In Section 3, we partially answer the question mentioned before and give an application of the main result to differential equations.

\section{Proof of the Main Theorem}

In this section, we prove Theorem 3. Note that there is an invertible matrix $P$ such that $P^{-1}\left(S_{2}-S_{1}\right) P$ is the Jordan normal form of $S_{2}-S_{1}$. Let $Q=P \oplus P \oplus P$. Then the condition that

$$
X=\left(\begin{array}{ccc}
I & I & I \\
S_{1} & S_{2} & S_{3} \\
S_{1}^{2} & S_{2}^{2} & S_{3}^{2}
\end{array}\right)
$$

is invertible is equivalent to that

$$
Q^{-1} X Q
$$

is invertible. So from the beginning, we can assume that $S_{2}-S_{1}$ is in Jordan normal form.

First we prove a lemma.

Lemma 4. Assume $S_{1}, S_{2} \in M_{n}(\mathbb{C})$ and the pair $\left(S_{1}, S_{2}\right)$ is an almost complete pair with $S_{2}-S_{1}=I_{n-1} \oplus 0$. Write

$$
S_{1}=\left(\begin{array}{ll}
A & \alpha \\
\beta & s
\end{array}\right),
$$

where $A \in M_{n-1}(\mathbb{C})$. Then $\alpha$ is not a zero vector. 
Proof. By some computations, we have

$$
S_{2}^{2}-S_{1}^{2}=\left(\begin{array}{cc}
2 A+I_{n-1} & \alpha \\
\beta & 0
\end{array}\right) \text {. }
$$

The last column of

$$
\left(\begin{array}{l}
S_{2}-S_{1} \\
S_{2}^{2}-S_{1}^{2}
\end{array}\right)
$$

is $(0,0, \ldots, 0, \alpha, 0)^{T}$, which is a $2 n$-dimensional column vector. By the definition of almost complete pair, $\alpha$ is not a zero vector.

Now we prove the main theorem.

Proof. From now on, we write $S_{1}=\left(\begin{array}{ccc}a_{11} & \cdots & a_{1 n} \\ \vdots & \ddots & \vdots \\ a_{n 1} & \cdots & a_{n n}\end{array}\right)$. We denote by $P(i, j) \in M_{n-1}(\mathbb{R})$ the matrix which is obtained by exchanging the columns of $i$ and $j$ of $(n-1) \times(n-1)$ identity matrix.

Without loss of generality, we can assume $S_{2}-S_{1}$ is in the Jordan normal form.

Case 1. Firstly, we consider the case that

$$
S_{2}-S_{1}=\left(\begin{array}{cc}
I_{n-1} & 0 \\
0 & 0
\end{array}\right)
$$

If $a_{1 n} \neq 0$, then we take

$$
S_{3}=S_{1}+\left(\begin{array}{cc}
0 & 0 \\
t I_{n-1} & 0
\end{array}\right)
$$

By some computation, the last columns of $S_{2}^{2}-S_{1}^{2}$ and $S_{3}^{2}-$ $S_{1}^{2}$ are $\left(a_{1 n}, a_{2 n}, \ldots, a_{n-1}, 0\right)^{T}$ and $\left(0, t a_{1 n}, t a_{2 n}, \ldots, t a_{n-1}\right)^{T}$, respectively, and the $i$ th $(1 \leq i \leq n-2)$ column of $S_{3}^{2}-S_{1}^{2}$ is

$$
\begin{aligned}
& \left(f_{i, 1}(t), f_{i, 2}(t), \ldots, f_{i, i+1}(t)\right. \\
& \left.\quad t^{2}+f_{i, i+2}(t), f_{i, i+3}(t), \ldots, f_{i, n}(t)\right)^{T}
\end{aligned}
$$

and the $(n-1)$ th column of $S_{3}^{2}-S_{1}^{2}$ is $\left(f_{n-1,1}(t), f_{n-1,2}(t), \ldots, f_{n-1, n}(t)\right)^{T}$, where $f_{i, 1}=t a_{1 i+1}$ and $f_{i, j}=t\left(a_{j i+1}+a_{j-1} i\right)(1 \leq i \leq n-1,2 \leq j \leq n)$. It is clear that

$$
\operatorname{det}\left(\begin{array}{ll}
S_{2}-S_{1} & S_{3}-S_{1} \\
S_{2}^{2}-S_{1}^{2} & S_{3}^{2}-S_{1}^{2}
\end{array}\right)
$$

is a polynomial in $t$. If $a_{1 n} \neq 0$, then the degree of this polynomial is $2 n-2$ and the leading term of this polynomial is $a_{1 n}^{2} t^{2 n-2}$. If $|t|$ is sufficiently large, we can get that

$$
X=\left(\begin{array}{ccc}
I & I & I \\
S_{1} & S_{2} & S_{3} \\
S_{1}^{2} & S_{2}^{2} & S_{3}^{2}
\end{array}\right)
$$

is invertible. If $a_{1 n}=0$, by Lemma 4 , then there must be some

$$
a_{\text {in }} \neq 0 \text {, }
$$

where $2 \leq i \leq n-1$. So

$$
\begin{aligned}
& \left(\begin{array}{cc}
P(1, i) & 0 \\
0 & 1
\end{array}\right)\left(\begin{array}{ll}
A & \alpha \\
\beta & 0
\end{array}\right)\left(\begin{array}{cc}
P(1, i) & 0 \\
0 & 1
\end{array}\right) \\
& =\left(\begin{array}{cc}
(P(1, i)) A P(1, i) & P(1, i) \alpha \\
\beta P & 0
\end{array}\right),
\end{aligned}
$$

where the first entry of the vector $P(1, i) \alpha$ is $a_{i n}$. Take $S_{3}$ such that

$$
(P(1, i) \oplus 1)\left(S_{3}-S_{1}\right)(P(1, i) \oplus 1)=\left(\begin{array}{cc}
0 & 0 \\
t I_{n-1} & 0
\end{array}\right) .
$$

Then

$$
X=\left(\begin{array}{ccc}
I & I & I \\
S_{1} & S_{2} & S_{3} \\
S_{1}^{2} & S_{2}^{2} & S_{3}^{2}
\end{array}\right)
$$

is invertible.

Case 2. Now we consider the case that $S_{2}-S_{1}$ has the Jordan normal form

$$
J\left(\lambda_{1}\right) \oplus J\left(\lambda_{2}\right) \oplus \cdots \oplus J\left(\lambda_{k}\right) \oplus 0,
$$

where $\lambda_{i} \neq 0,1 \leq i \leq k$. If $a_{1 n} \neq 0$, then we take

$$
S_{3}=S_{1}+\left(\begin{array}{cc}
0 & 0 \\
t I_{n-1} & 0
\end{array}\right)
$$

If $a_{1 n}=0$ and $a_{i n} \neq 0(1<i<n)$, then we take $S_{3}$ such that

$$
(P(1, i) \oplus 1)\left(S_{3}-S_{1}\right)(P(1, i) \oplus 1)=\left(\begin{array}{cc}
0 & 0 \\
t I_{n-1} & 0
\end{array}\right) .
$$

In any case when $|t|$ is sufficiently large, we can get that

$$
X=\left(\begin{array}{ccc}
I & I & I \\
S_{1} & S_{2} & S_{3} \\
S_{1}^{2} & S_{2}^{2} & S_{3}^{2}
\end{array}\right)
$$

is invertible.

Case 3. Now we consider the case that 0 is not a single root of characteristic polynomial of $S_{2}-S_{1}$; that is, $S_{2}-S_{1}$ has the Jordan normal form:

$$
J\left(\lambda_{1}\right) \oplus J\left(\lambda_{2}\right) \oplus \cdots \oplus J\left(\lambda_{s}\right) \oplus J(0) .
$$

If $n=2$, then

$$
S_{2}-S_{1}=\left(\begin{array}{ll}
0 & 0 \\
1 & 0
\end{array}\right)
$$


By Lemma $4, a_{12} \neq 0$. Take

$$
S_{3}=S_{1}+\left(\begin{array}{ll}
1 & 0 \\
0 & 0
\end{array}\right)
$$

Then

$$
\operatorname{det}\left(\begin{array}{ccc}
I & I & I \\
S_{1} & S_{2} & S_{3} \\
S_{1}^{2} & S_{2}^{2} & S_{3}^{2}
\end{array}\right)=\operatorname{det}\left(\begin{array}{cc}
S_{2}-S_{1} & S_{3}-S_{1} \\
S_{2}^{2}-S_{1}^{2} & S_{3}^{2}-S_{1}^{2}
\end{array}\right)=-t^{2} a_{12}^{2} \neq 0 .
$$

We can conclude that

$$
X=\left(\begin{array}{ccc}
I & I & I \\
S_{1} & S_{2} & S_{3} \\
S_{1}^{2} & S_{2}^{2} & S_{3}^{2}
\end{array}\right)
$$

is invertible.

Firstly, we prove the case that the normal form itself is

$$
\left(\begin{array}{cc}
0 & 0 \\
t I_{n-1} & 0
\end{array}\right)
$$

If $a_{1 n} \neq 0$, then we take

$$
S_{3}=S_{1}+\left(\begin{array}{ccccccc}
t & 0 & 0 & 0 & \cdots & 0 & t \\
0 & 0 & 0 & 0 & \cdots & 0 & 0 \\
t & t & 0 & 0 & \cdots & 0 & 0 \\
0 & 0 & t & 0 & \cdots & 0 & 0 \\
\vdots & \vdots & \vdots & \ddots & & \vdots & \vdots \\
0 & 0 & 0 & 0 & \cdots & t & 0
\end{array}\right)
$$

So

$$
\left(S_{3}-S_{1}\right)^{2}=\left(\begin{array}{ccccccc}
t^{2} & 0 & 0 & \cdots & 0 & t^{2} & 0 \\
0 & 0 & 0 & \cdots & 0 & 0 & 0 \\
t^{2} & 0 & 0 & \cdots & 0 & 0 & 0 \\
0 & t^{2} & 0 & \cdots & 0 & 0 & 0 \\
\vdots & \vdots & \ddots & & \vdots & \vdots & \vdots \\
0 & 0 & 0 & \cdots & t^{2} & 0 & 0
\end{array}\right)
$$

By some computation,

$$
\operatorname{det} X=\operatorname{det}\left(\begin{array}{ccc}
I & I & I \\
S_{1} & S_{2} & S_{3} \\
S_{1}^{2} & S_{2}^{2} & S_{3}^{2}
\end{array}\right)
$$

is a polynomial in $t$, where the leading term of it is $a_{1 n} t^{2 n-1}$. So if $a_{1 n} \neq 0$, then $X$ is invertible when $|t|$ is sufficiently large. If $a_{1 n}=0$, then there must be an $i \in\{2,3, \ldots, n-1\}$ such that $a_{\text {in }} \neq 0$. If $i \in\{2,3, \ldots, n-2\}$, take $S_{3}$ such that

$$
\begin{gathered}
(P(1, i-1) \oplus 1)\left(S_{3}-S_{1}\right)(P(1, i-1) \oplus 1) \\
\quad=\left(\begin{array}{ccccccc}
t & 0 & 0 & 0 & \cdots & 0 & t \\
0 & 0 & 0 & 0 & \cdots & 0 & 0 \\
t & t & 0 & 0 & \cdots & 0 & 0 \\
0 & 0 & t & 0 & \cdots & 0 & 0 \\
\vdots & \vdots & \vdots & \ddots & & \vdots & \vdots \\
0 & 0 & 0 & 0 & \cdots & t & 0
\end{array}\right)
\end{gathered}
$$

When $|t|$ is sufficiently large, we have $X$ being invertible. If $i=n-1$, that is to say, the last column of $S_{2}^{2}-S_{1}^{2}$ is $\left(0,0, \ldots, 0, a_{n-1}\right)^{T}$, then we take

$$
S_{3}=S_{1}+\left(\begin{array}{ccccccc}
t & 0 & 0 & 0 & \cdots & 1 & 0 \\
0 & t & 0 & 0 & \cdots & 0 & 0 \\
0 & 0 & t & 0 & \cdots & 0 & 0 \\
0 & 0 & 0 & t & \cdots & 0 & 0 \\
\vdots & \vdots & \vdots & \vdots & \ddots & \vdots & \vdots \\
0 & 0 & 0 & 0 & \cdots & t & 0 \\
0 & 0 & 0 & 0 & \cdots & 0 & 0
\end{array}\right)
$$

We have

$$
\left(S_{3}-S_{1}\right)^{2}=\left(\begin{array}{ccccccc}
t^{2} & 0 & 0 & 0 & \cdots & 2 t & 0 \\
0 & t^{2} & 0 & 0 & \cdots & 0 & 0 \\
0 & 0 & t^{2} & 0 & \cdots & 0 & 0 \\
0 & 0 & 0 & t^{2} & \cdots & 0 & 0 \\
\vdots & \vdots & \vdots & \vdots & \ddots & \vdots & \vdots \\
0 & 0 & 0 & 0 & \cdots & t^{2} & 0 \\
0 & 0 & 0 & 0 & \cdots & 0 & 0
\end{array}\right)
$$

where the first entry of the last column of $S_{3}^{2}-S_{1}^{2}$ is $a_{n-1}$. So the leading term of the polynomial

$$
\operatorname{det} X=\operatorname{det}\left(\begin{array}{ccc}
I & I & I \\
S_{1} & S_{2} & S_{3} \\
S_{1}^{2} & S_{2}^{2} & S_{3}^{2}
\end{array}\right)
$$

is $a_{n-1 n}^{2} t^{2 n-3}$. Thus

$$
X=\left(\begin{array}{ccc}
I & I & I \\
S_{1} & S_{2} & S_{3} \\
S_{1}^{2} & S_{2}^{2} & S_{3}^{2}
\end{array}\right)
$$

is invertible when $|t|$ is sufficiently large.

Now we prove the general case that $S_{2}-S_{1}$ has the Jordan normal form:

$$
J\left(\lambda_{1}\right) \oplus J\left(\lambda_{2}\right) \oplus \cdots \oplus J\left(\lambda_{s}\right) \oplus J(0),
$$

where $\lambda_{i} \neq 0,1 \leq i \leq s$. If there is some $a_{n i} \neq 0, m+1 \leq i \leq n$, we assume that $J(0)$ is a matrix of order $n-m$. For any square matrix $B$ of order $n-m$, we can construct a square matrix $A$ of order $n-m$ such that

$$
\left(\begin{array}{cc}
J(0) & A \\
(J(0)+B)^{2}-J^{2}(0) & (A+B)^{2}-A^{2}
\end{array}\right)
$$

is invertible. Take

$$
S_{3}=S_{1}+\left(t I_{n-m} \oplus A\right) .
$$

If $a_{\text {in }}=0$, for all $m+1 \leq i \leq n$, there must be some $j$ such that $1 \leq j \leq m, a_{j n} \neq 0$. Let $Y=X P$, where $P$ is the matrix which is obtained by exchanging the columns $m+1$ and $n$ of $n \times n$ identity matrix. Then $\operatorname{det} X=-\operatorname{det} Y$ and

$$
Y=J\left(\lambda_{1}\right) \oplus J\left(\lambda_{2}\right) \oplus \cdots \oplus J\left(\lambda_{i}\right) \oplus 0 \oplus C,
$$


where the square matrix $C$ of order $n-m+1$ has the form

$$
\left(\begin{array}{cc}
0 & 1 \\
I_{n-m} & 0
\end{array}\right)
$$

Let $T$ be a matrix such that $T^{-1} C T$ has the Jordan normal form $J$. Then we can consider the matrix $Z=\left(I \oplus T^{-1}\right) Y(I \oplus T)$. Note that $Z$ has the form

$$
Z=J\left(\lambda_{1}\right) \oplus J\left(\lambda_{2}\right) \oplus \cdots \oplus J\left(\lambda_{i}\right) \oplus 0 \oplus J .
$$

Denote $S=J\left(\lambda_{1}\right) \oplus J\left(\lambda_{2}\right) \oplus \cdots \oplus J\left(\lambda_{i}\right) \oplus 0$. For any square matrix $B$, we can construct a square matrix $A$ of order $m+1$ such that

$$
\left(\begin{array}{cc}
S & A \\
(S+B)^{2}-S^{2} & (A+B)^{2}-A^{2}
\end{array}\right)
$$

is invertible. Take $S_{3}=S_{1}+A \oplus t I_{m-1}$. Then we can conclude that

$$
\left(\begin{array}{ccc}
I & I & I \\
S_{1} & S_{2} & S_{3} \\
S_{1}^{2} & S_{2}^{2} & S_{3}^{2}
\end{array}\right)
$$

is invertible. The proof is complete.

\section{Applications}

For given monic matrix polynomials $L_{1}(\lambda), L_{2}(\lambda), \ldots, L_{n}(\lambda)$, the construction of a common multiple of them was presented in [2].

Suppose that $\left(S_{1}, S_{2}\right)$ is an almost complete pair. By Theorem 3 in Section 1 , we can find $S_{3} \in M_{n}(\mathbb{C})$ such that $\left(S_{1}, S_{2}, S_{3}\right)$ is a complete triple; that is,

$$
\left(\begin{array}{ccc}
I & I & I \\
S_{1} & S_{2} & S_{3} \\
S_{1}^{2} & S_{2}^{2} & S_{3}^{2}
\end{array}\right)
$$

is invertible. Let

$$
W_{m}\left(S_{1}, S_{2}, S_{3}\right)=\left(\begin{array}{ccc}
I & I & I \\
S_{1} & S_{2} & S_{3} \\
\vdots & \vdots & \vdots \\
S_{1}^{m-1} & S_{2}^{m-1} & S_{3}^{m-1}
\end{array}\right) .
$$

Then we can conclude that

$$
\min \left\{m \geq 1 \mid \operatorname{Ker} W_{m}=\operatorname{Ker} W_{m+1}\right\}=3 .
$$

By Theorem 9.11 of [2], there exists a monic matrix polynomial $L(\lambda)$ with degree 3 which is a common multiple of $I \lambda-S_{1}, I \lambda-S_{2}, I \lambda-S_{3}$.

As is well known, spectral method for the analysis of monic matrix polynomials was important (cf. [6-13]). The spectral method basically uses the construction of standard pairs and standard triples (e.g., $[14,15])$ to solve differential equations.

Now we recall definitions of standard pair and standard triple for monic polynomials.
Definition 5 (see [2]). A pair of matrices $(X, T)$, where $X$ is $n \times$ $n l$ and $T$ is $n l \times n l$, is called a standard pair for the monic matrix polynomial $L(\lambda)$ if the following conditions are satisfied:

(i) $\operatorname{col}\left(X T^{i}\right)_{i=0}^{l-1}$ is nonsingular, where

$$
\operatorname{col}\left(X T^{i}\right)_{i=0}^{l-1}=\left(\begin{array}{c}
X \\
X T \\
\vdots \\
X T^{l-1}
\end{array}\right)
$$

(ii) $\sum_{i=0}^{l-1} A_{i} X T^{i}+X T^{l}=0$.

If $T$ is in Jordan normal form, we call $(X, T)$ a Jordan pair for the monic matrix polynomial $L(\lambda)$.

Definition 6 (see [2]). A triple of matrices $(X, T, Y)$, where $X$ is $n \times n l, T$ is $n l \times n l$, and $Y$ is $n l \times n$, is called a standard triple for the monic matrix polynomial $L(\lambda)$ if $(X, T)$ is a standard pair for $L(\lambda)$, and $Y=\left(\begin{array}{c}X \\ X T \\ \vdots \\ X T^{l-1}\end{array}\right)^{-1}\left(\begin{array}{c}0 \\ \vdots \\ 0 \\ I\end{array}\right)$.

Now we recall the explicit formulas for solutions of the following differential equation:

$$
L\left(\frac{d}{d t}\right) x(t)=\frac{d^{l} x(t)}{d t^{l}}+\sum_{j=0}^{l-1} A_{j} \frac{d^{j} x(t)}{d t^{j}}=f(t) .
$$

Lemma 7 (Theorem 2.9 in [2]). The general solution of (60) is given by the formula

$$
x(t)=X e^{t T} c+X \int_{a}^{t} e^{(t-s) T} Y f(s) d s, \quad t \in[a, b],
$$

where $(X, T, Y)$ is a standard triple of $L(\lambda)$ and $c \in \mathbb{C}^{n l}$ is arbitrary. In particular, the general solution of the homogeneous equation

$$
L\left(\frac{d}{d t}\right) x(t)=0
$$

is given by the formula

$$
x(t)=X e^{t T} c, \quad c \in \mathbb{C}^{n l}
$$

Before we give the main result of this section, we introduce some notations. Suppose

$$
J_{1}=X_{1}^{-1} S_{1} X_{1}, \quad J_{2}=X_{2}^{-1} S_{2} X_{2}, \quad J_{3}=X_{3}^{-1} S_{3} X_{3}
$$

are the Jordan forms of $S_{1}, S_{2}, S_{3}$, respectively. Write

$$
X=\left(\begin{array}{lll}
X_{1} & X_{2} & X_{3}
\end{array}\right), \quad J=J_{1} \oplus J_{2} \oplus J_{3} .
$$

Theorem 8. Let $\left(S_{1}, S_{2}, S_{3}\right)$ be a complete triple of $L(\lambda)=0$, where $L(\lambda)=I \lambda^{3}+A_{2} \lambda^{2}+A_{1} \lambda+A_{0}$. Then every solution of

$$
L\left(\frac{d}{d t}\right) u=f
$$


is given by

$$
\begin{aligned}
u(t)= & e^{S_{1} t} c_{1}+e^{S_{2} t} c_{2}+e^{S_{3} t} c_{3} \\
& +X \int_{a}^{t} e^{(t-s) J}\left(\begin{array}{c}
Y_{1} \\
Y_{2} \\
Y_{3}
\end{array}\right) f(s) d s, \quad t \in[a, b],
\end{aligned}
$$

for some $c_{1}, c_{2}, c_{3} \in \mathbb{C}^{n}$, where

$$
\begin{aligned}
\left(\begin{array}{c}
Y_{2} \\
Y_{3}
\end{array}\right) & =\left(\begin{array}{ll}
S_{2}-S_{1} & S_{3}-S_{1} \\
S_{2}^{2}-S_{1}^{2} & S_{3}^{2}-S_{1}^{2}
\end{array}\right)^{-1}\left(\begin{array}{c}
0 \\
X_{3}^{-1}
\end{array}\right), \\
Y_{1} & =-X_{1}^{-1} X_{2} Y_{2}-X_{1}^{-1} X_{3} Y_{3} .
\end{aligned}
$$

In particular, every solution of

$$
L\left(\frac{d}{d t}\right) u=0
$$

has the form

$$
u(t)=e^{S_{1} t} c_{1}+e^{S_{2} t} c_{2}+e^{S_{3} t} c_{3}
$$

where $c_{1}, c_{2}, c_{3} \in \mathbb{C}^{n}$.

Proof. Using Lemma 7, we take

$$
Y=\left(\begin{array}{c}
Y_{1} \\
Y_{2} \\
Y_{3}
\end{array}\right)=X^{-1}\left(\begin{array}{ccc}
I & I & I \\
S_{1} & S_{2} & S_{3} \\
S_{1}^{2} & S_{2}^{2} & S_{3}^{2}
\end{array}\right)^{-1}\left(\begin{array}{l}
0 \\
0 \\
I
\end{array}\right)
$$

Then $(X, J, Y)$ is a standard triple; it is easy to get the conclusion.

Remark 9. The above theorem and its proof are motivated by those in [5] (cf. also Sections 2.4 and 2.5 in [2]).

\section{Conflict of Interests}

The authors declare that there is no conflict of interests regarding the publication of this paper.

\section{Acknowledgments}

The authors would like to thank the referees for their helpful comments and suggestions. The research was supported by National Natural Science Foundation of China (Grant no. 11001064), by the Fundamental Research Funds for the Central Universities (Grant no. HIT. NSRIF. 2014085), and by the Scientific Research Foundation for the Returned Overseas Chinese Scholars.

\section{References}

[1] I. Gohberg, M. A. Kaashoek, L. Lerer, and L. Rodman, "Common multiples and common divisors of matrix polynomialsII. Vandermonde and resultant matrices," Linear and Multilinear Algebra, vol. 12, no. 3, pp. 159-203, 1983.
[2] I. Gohberg, P. Lancaster, and R. Rodman, Matrix Polynomails, Academic Press, London, UK, 1982.

[3] M. G. Krein, H. Langer, and R. Troelstra, "On some mathematical principles in the linear theory of damped oscillations of continua I," Integral Equations and Operator Theory, vol. 1, no. 3, pp. 364-399, 1978.

[4] M. G. Krein and H. Langer, "On some mathematical principles in the linear theory of damped oscilations of continua II," Integral Equations and Operator Theory, vol. 1, no. 4, pp. 539$566,1978$.

[5] P. Lancaster, "A fundamental theorem on lambda-matrices with applications-I. Ordinary differential equations with constant coefficients," Linear Algebra and Its Applications, vol. 18, no. 3, pp. 189-211, 1977.

[6] J. A. Ball, I. Gohberg, and L. Rodman, "Common minimal multiples and divisors for rational matrix functions," Linear Algebra and Its Applications, vol. 137-138, pp. 621-662, 1990.

[7] N. Cohen, "Spectral analysis of regular matrix polynomials," Integral Equations and Operator Theory, vol. 6, no. 1, pp. 161-183, 1983.

[8] I. Gohberg, M. A. Kaashoek, and L. Rodman, "Spectral analysis of families of operator polynomials and a generalized Vandermonde matrix-II: the infinite dimensional case," Journal of Functional Analysis, vol. 30, no. 3, pp. 358-389, 1978.

[9] I. Gohberg, P. Lancaster, and L. Rodman, "Spectral analysis of matrix polynomials-I. Canonical forms and divisors," Linear Algebra and Its Applications, vol. 20, no. 1, pp. 1-44, 1978.

[10] I. Gohberg, P. Lancaster, and L. Rodman, "Spectral analysis of matrix polynomials-II. The resolvent form and spectral divisors," Linear Algebra and Its Applications, vol. 21, no. 1, pp. 65-88, 1978.

[11] I. Gohberg, P. Lancaster, and R. Rodman, "Representations and divisibility of operator polynomials," Canadian Journal of Mathematics, vol. 30, pp. 1045-1069, 1978.

[12] M. A. Kaashoek, "Recent developments in the spectral analysis of matrix and operator polynomials," in Proceedings of the Bicentennial Congress Wiskundig Genootschap, pp. 233-247, Math. Centre Tracts, 101, Mathematisch Centrum, Amsterdam, The Netherlands, 1979.

[13] J. Maroulas, "Factorization of matrix polynomials with multiple roots," Linear Algebra and Its Applications, vol. 69, pp. 9-32, 1985.

[14] M. Al-Ammari and F. Tisseur, "Standard triples of structured matrix polynomials," Linear Algebra and Its Applications, vol. 437, no. 3, pp. 817-834, 2012.

[15] P. Lancaster, U. Prells, and L. Rodman, "Canonical structures for palindromic matrix polynomials," Operators and Matrices, vol. 1, no. 4, pp. 469-489, 2007. 


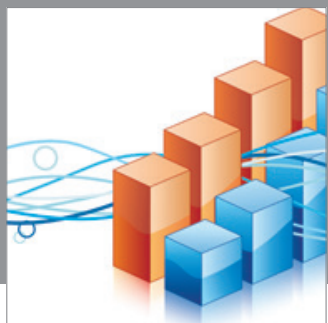

Advances in

Operations Research

mansans

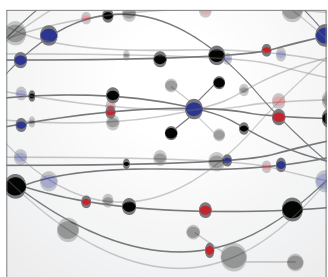

The Scientific World Journal
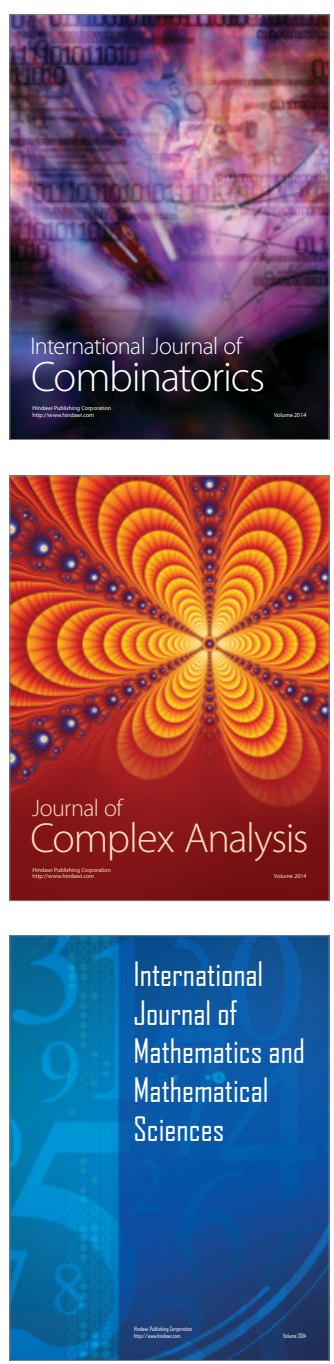
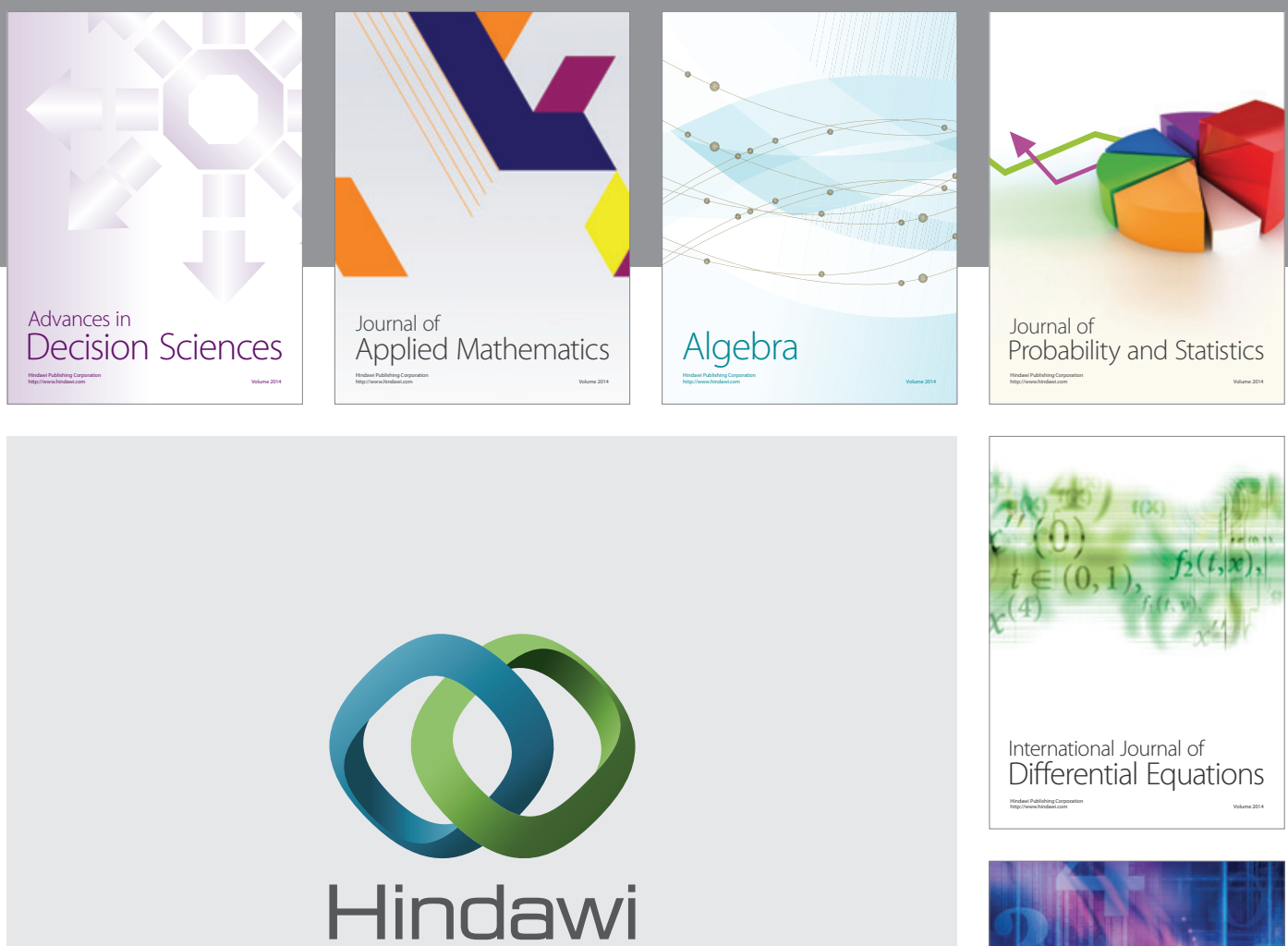

Submit your manuscripts at http://www.hindawi.com
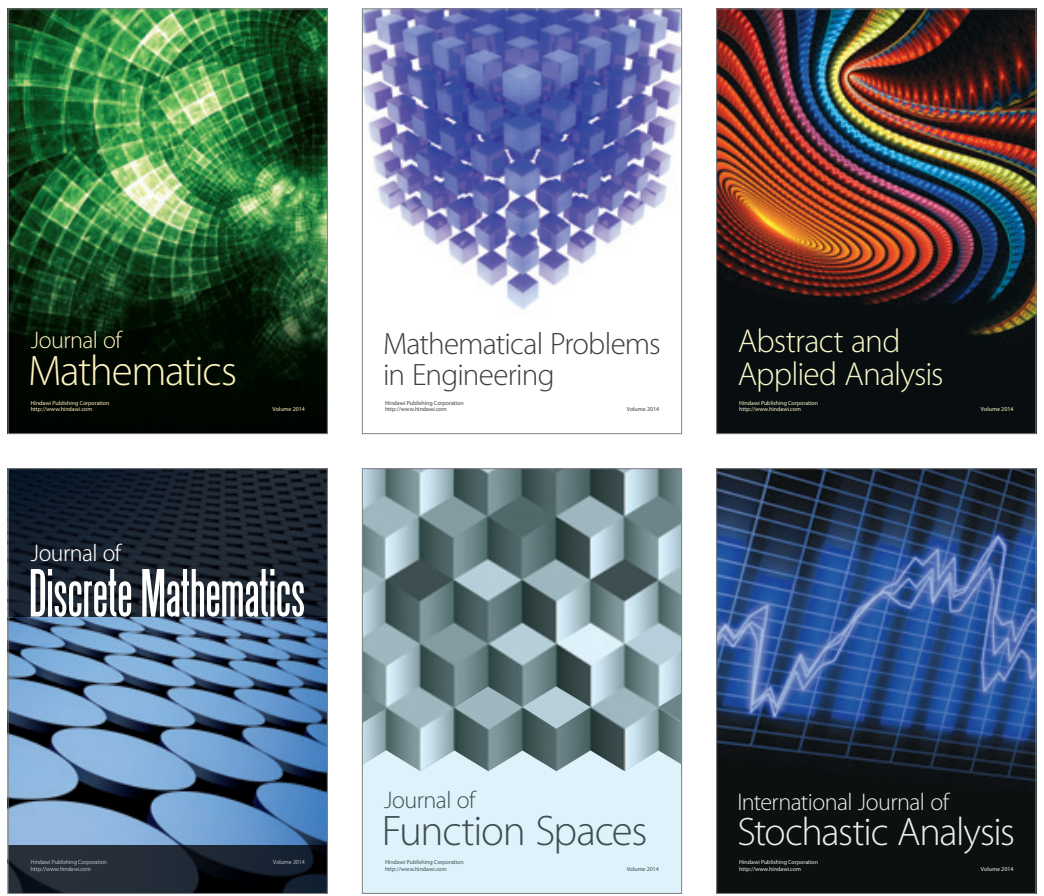

Journal of

Function Spaces

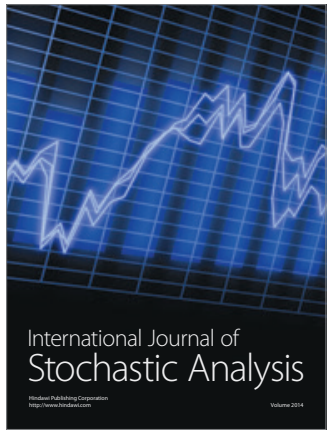

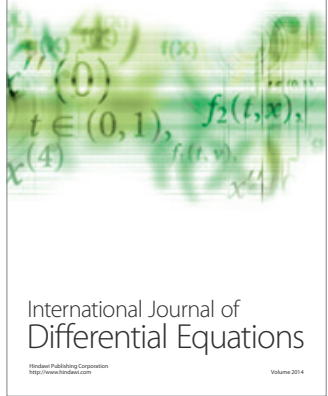
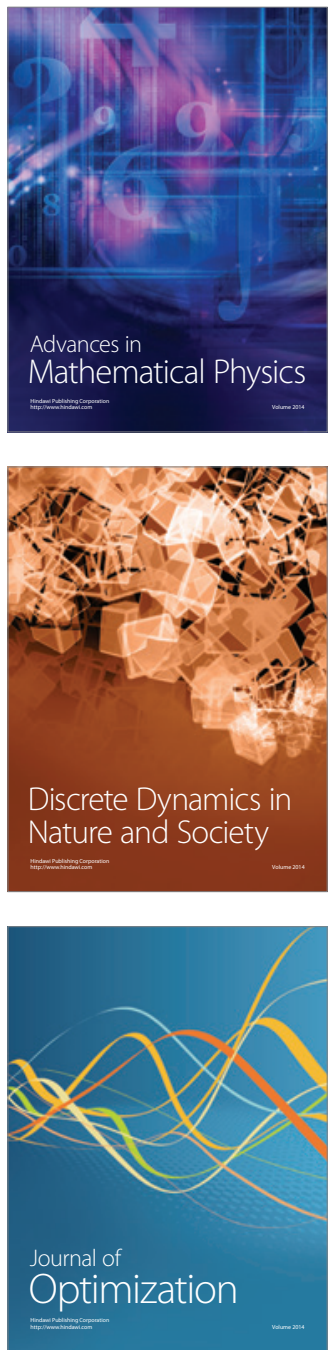